\title{
Jin Fu Kang
}

National Cancer Institute

\section{Source}

National Cancer Institute. lin Fu Kang. NCI Thesaurus. Code C61316.

A traditional Chinese herbal medicine derived from the plant Astragalus membranaceus with potential immunopotentiation activity. Jin Fu Kang may stimulate anti-tumor macrophage and natural killer cell activity and may enhance immune recognition of tumor cells by inhibiting the production of T-helper cell type 2 (Th2) cytokines such as interleukin-4 (IL-4) and interleukin-10 (IL-10). 